\title{
GÊNERO PHYSALIS - UMA REVISÃO SOBRE VITAESTERÓIDES
}

Therezinha C. B. Tomassini, Nancy S. Barbi, Ivone M. Ribeiro, Deise C. D. Xavier

Far-Manguinhos/FIOCRUZ - Rua Sizenando Nabuco, 100 - Manguinhos - 21041-250 - Rio de Janeiro - RJ

Recebido em 1/6/98; aceito em 25/6/99

\begin{abstract}
GENUS PHYSALIS - A REVISION OF WITHASTEROIDS. This review describes results on the chemistry and spectroscopic data of some ergostane derivatives named withasteroids which have been obtained from Physalis genus. The main aim of this report is concerned with the description and characterization of Physalins. These natural product substances are $\mathrm{C28}$ seco steroid lactone type compounds that have been shown biological activities against human illness such as immunodeficiency, neoplasic tumors, inflammatory process and tropical endemic diseases. Physalins appear to be a source for new drugs to be apply as medicine.
\end{abstract}

Keywords: Physalis; Physalins; Withasteroids.

\section{INTRODUÇÃO}

Os vitaesteróides são substâncias que reproduzem o esqueleto intacto ou modificado do ergostano ${ }^{1-4}$. Estes derivados do ergostano são constituintes polioxigenados presentes, preponderantemente, nas espécies de Solanaceae. Tal ocorrência, porém, não se limita àquela família, como a princípio alguns pesquisadores faziam $\mathrm{crer}^{4}$, já que este grupo de substâncias também pode ser encontrado nas Leguminoseae $^{5,6}$ e Taccacedae ${ }^{7}$. A família Solanaceae inclui uma ampla variedade de plantas que são econômica e farmacologicamente importantes.

\section{- Características e classificação dos vitaesteróides}

Os vitaesteróides possuem função lactônica em C-26, fornecendo uma variedade de estruturas classificadas em oito grupos, cujos os esqueletos básicos encontram-se representados pelas estruturas de 1 a 8 (Fig. 1).

- vitanolidos (1)

- vitanolidos "modificados" (anéis $\underline{\mathrm{A}}$ ou $\underline{\mathrm{D}}$ aromatizados 2,3)

- vitafisalinas (4)

- acnistinas (5)

- ixocarpalactonas (6)

- perulactonas (7)

- fisalinas (8)

Investigações mais recentes visam esclarecer as relações biogenéticas ${ }^{8,9}$ entre os grupos acima mencionados. Tais correlações implicam em acentuadas modificações nos esqueletos carbocíclios, na cadeia lateral ou em ambos. Como exemplos, citam-se os aroamtizados nos anéis $\underline{\mathrm{A}}$ e $\underline{\mathrm{D}}$, os epóxi-lactóis, dentre outros.

Aqueles oito grupos estruturais encontram- se distribuídos nos gêneros abaixo, pertencentes a família Solanaceae.
- Acnistus
- Nicandra
- Datura
- Physalis
- Deprea
- Dunalia
- Iochroma
- Jaborosa
- Lycium
- Salpichroa
- Trechonates
- Tubocapsicum
- Withania
- Witheringia

Observa-se uma grande diversificação estrutural de derivados ergostânicos nestes gêneros cabendo a Withania e Physalis a maior produção de vitanolídos e fisalinas respectivamente ${ }^{3}$.

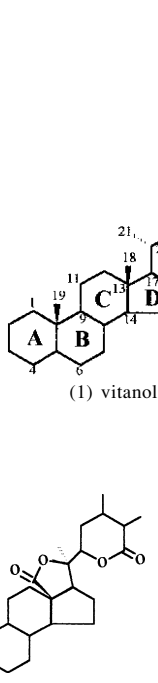

(4) vitafisalina

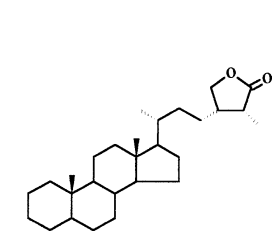

(7) perulactona

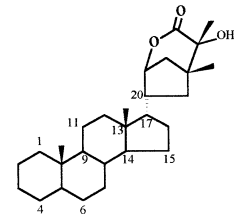

(5) acnistina

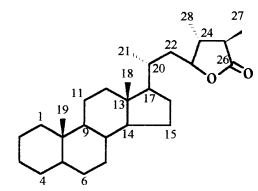

(6) ixocarpalactona

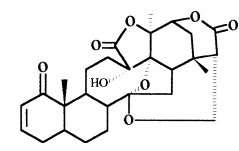

(8) fisalina

Figura 1. Esqueletos básicos de vitaesteróides.

Witaferina $\underline{\mathrm{A}}(9)$, o primeiro vitanolído isolado de Withania sonnifera, teve sua estrutura totalmente elucidada por Lavie e colaboradores, em $1965^{10}$, seguindo-se várias outras descobertas, descrições de diferentes e diversificados tipos deste grupo de esteróides, provindos da família Solanaceae ${ }^{11 a, b}$

\section{- Vitanolídos}

Dentre os vitaesteróides representados por seus esqueletos básicos (1 a 8), os vitanolídos são os mais abundantes, sendo normalmente mencionados como precursores das vitafisalinas e acnistinas.

Subdividem-se em duas classes principais de acordo com a 
orientação $(\alpha$ ou $\beta)$ da cadeia lateral, fixa no $C$ - 17 . Os $\alpha$ vitanolídos descritos são em número de trinta e seis enquanto que os $\beta$, bem mais freqüentes, atingem o número de cento e trinta e duas substâncias, com estruturas já totalmente elucidadas ${ }^{3}$.

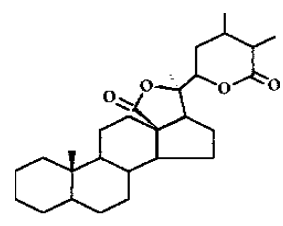

(9) witaferina A de um bom grupo de saída no C-21, segundo ilustrado no esquema $2^{3}$.

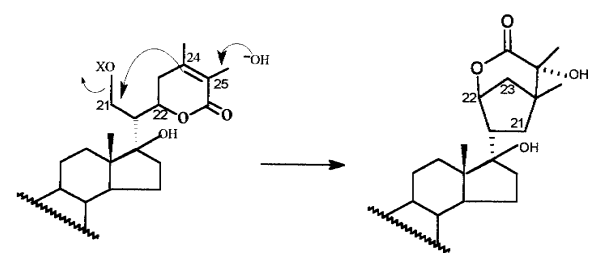

Esquema 2. Provável mecanismo para formação da cadeia bicíclica de acnistinas.

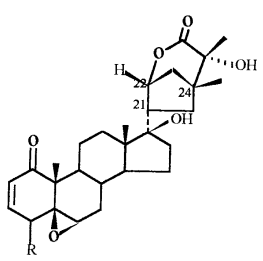

(15) acnistina $\mathrm{A}, \mathrm{R}=\mathrm{H}^{(17)}$ um lactol ou uma lactona de cinco membros ligada ao anel D. A seguir encontram-se alguns exemplos de representantes deste grupo:

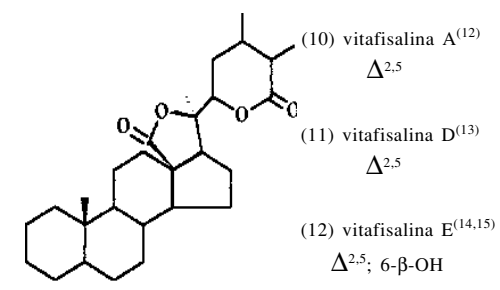

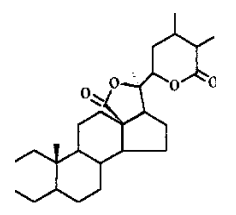

(13) vitafisalina $B^{(12)}$

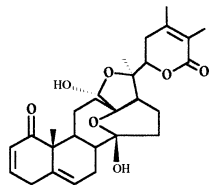

(14) vitafisalina $\mathrm{C}^{(16)}$
A formação da vitafisalina $\underline{C}(14)$ segue o mecanismo mostrado no Esquema 1.

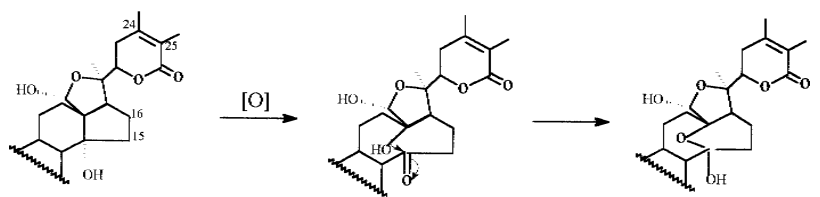

Esquema 1. Provável mecanismo para formação de ponte oxirânica de vitafisalina $C$, segundo Glotter ${ }^{2}$.

A relação biogenética destas com as fisalinas é contraditória uma vez que as vitafisalinas prescindem da função carbonilada em C-15, essencial para a formação do anel E, originando a ligação C-16 e C-24 nas fisalinas.

Outro pressuposto de que as vitafisalinas são precursores das fisalinas encontra suporte no fato da vitafisalina $\underline{C}$ (14) ter uma hidroxila em C-14 e possuir uma ponte oxirânica, conforme estrutura da fisalina $\underline{\mathrm{A}}(22)$.

\section{- Acnistinas}

As acnistinas são vitaesteróides com cadeia lateral bicíclica inserida em C-17. A formação de uma nova ligação C-21, C27 ocorre, devido a reação tipo $\mathrm{SN}_{2}$, em vitanolídos portadores

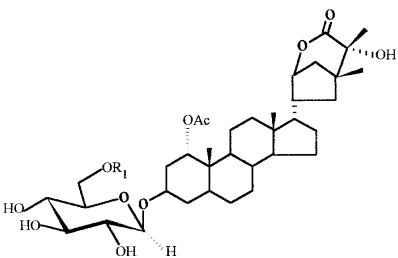

(17) tubocaposído $\mathrm{A}, \mathrm{R} 1=\mathrm{G} 1^{(18)}$

Segundo Glotter ${ }^{19}$ as acnistinas $\underline{A}$ e $\underline{E}$ (15 e 16) são derivadas de um precursor possuindo as características de uma $\delta$ lactona $\alpha-\beta$ insaturada cujo carbono 20 está orientado em posição $\alpha$. A hidroxilação do grupo metila, em C-21, seguida de transformação deste em um bom grupo de saída possibilita o ataque nucleofílico do sistema $\pi$ da ligação dupla, C-24 C-25, levando ao fechamento do novo anel ciclopentânico nas acnistinas (15 e 16)

\section{- Ixocarpalactonas}

As alterações devidas ao grupo hidroxila em C-22 dão origem a vitaesteróides diferenciados que apresentam anel $\gamma$ lactônico saturado e com orientação do tipo $\beta$ (18 e 19).

Tais características podem ser observadas nas ixocarpalactonas abaixo representadas,

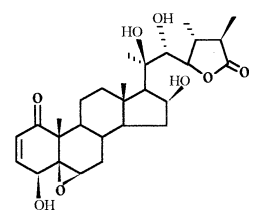

(18) ixocarpalactona $\mathrm{A}^{(20)}$

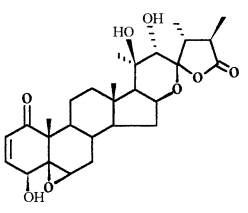

(19) ixocarpalactona $\mathrm{B}^{(21)}$
A ixocarpalactona $\underline{B}$ (19) tem estrutura do tipo espirocetal e sua formação parece ser devido a oxidação da hidroxila em C22 gerando a cetona correspondente.

\section{- Perulactonas}

São relacionadas aos vitaesteróides cujo C-28 é oxidado a álcool primário. Perulactonas $\underline{A}^{22}$ e $\underline{B}^{23}(20$ e 21$)$, isoladas de 
Physalis peruviana, ilustram a lactonização do derivado ciclizado em C-22 e C-26.

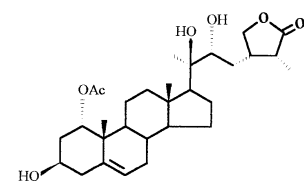

(20) perulactona A

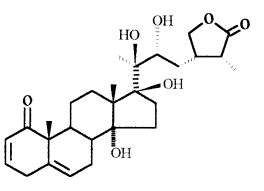

(21) perulactona B

\section{- Fisalinas}

A presente revisão enfatiza os vitaesteróides encontrados no gênero Physalis, fornecedor das fisalinas (22 a 41), cujas espécies classificam-se em:

- P. alkekengi

- P. alkekengi var francheti

- P. angulata

- P. Ixocarpa

- P. lanifolia

- P. minima

- P. peruviana

- P. phyladelphia

- P. pubescens

- P. viscosa

Destas já foram extraídas dezenove fisalinas.

Essas espécies não se limitam a produzir fisalinas, elaborando também, em sua biogênese os vitanolídos, vitafisalinas entre outros.

As fisalinas são moléculas de estruturas bastante complexas, pois além da lactona apresentam uma outra $\gamma$ lactona fundida ao anel D. São derivados esteroidais do tipo 13,14-seco16,24 ciclo ergostano, carbonilados em C-15.

A junção C-16 e C-24 parece ser formada pela reação de adição de Michael intramolecular, sendo mais apropriado sugerir que a ciclização é resultante da reação $\mathrm{SN}_{2}$ em $\mathrm{C}-27$, nos vitanolídos com um bom grupo de saída, naquela posição. $\mathrm{O}$ carbanion em C-16 atacaria o centro eletro deficiente em C-24 com simultânea migração da dupla ligação, eliminação do grupo de saída em C-27, formando a $\delta$ lactona $\gamma$ metilênica e por conseqüência a nova ligação C-16 e C-24, conforme pode ser ilustrado no Esquema 3:

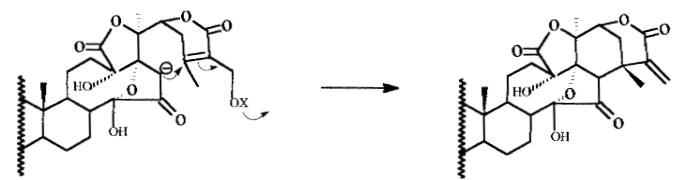

Esquema 3. Provável mecanismo da formação da ligação C-16 C-24 nas fisalinas ${ }^{3}$.

A ruptura para obtenção dos seco-derivados seria resultante da clivagem oxidativa efetuada por agente oxidante biológico, com o grupo $\mathrm{OH}$ em $\mathrm{C}-14$ tendo a carbonila em $\mathrm{C}-15$ como agente facilitador 18,24 .

Alguns vitaesteróides insaturados em $\Delta^{16}$ com substituintes oxigenados em C-14 e C-15 são considerados representantes ideais para o rompimento da ligação C-13,C-14. A ocorrência daqueles derivados $\Delta^{16}$, nas plantas ricas em fisalinas, leva a acreditar que eles sejam tanto os precursores quanto os desencadeadores desta complexa etapa da biogênese.

É interessante notar a pluralidade de anéis que este grupo de vitaesteróides apresenta. Das dezenove já elucidadas, cinco são octacíclicas, oito nonacíclicas, cinco decacíclicas e uma undecacíclica.

As três lactonas presentes na fisalina $\underline{P}$ (38) são relatadas como provenientes de um possível rearranjo, em sua molécula, do tipo ácido benzílico ${ }^{25,20}$.

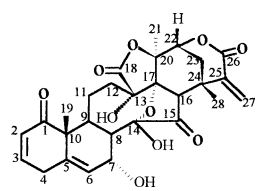

(22) fisalina $A^{(27,28)}$

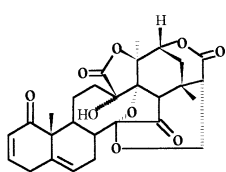

(23) fisalina $B^{(27)}$

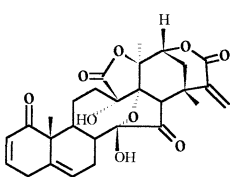

(24) fisalina $\mathrm{C}^{(29)}$

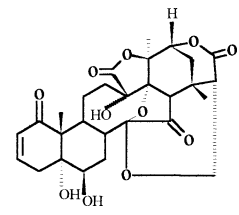

(25) fisalina $D^{(30,31)}$

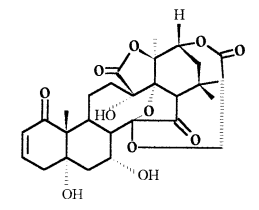

(26) fisalina $\underline{\underline{E}}^{(33)}$
A estrutura da "fisalina E" (26) foi recentemente contestada por Kawai et al. ${ }^{33}$, os quais afirmaram serem os dados apresentados por Raw ${ }^{31}$ idênticos aos da fisalina $\mathrm{D}(25),(5 \alpha$, $6 \beta$ - diidroxifisalina).

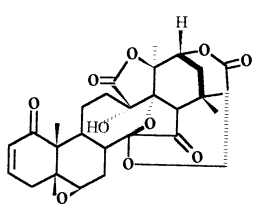

(27) fisalina $\underline{\mathrm{F}}^{(31)}$

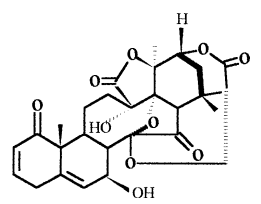

(29) "fisalina $\underline{\mathrm{H}}^{(30)}$

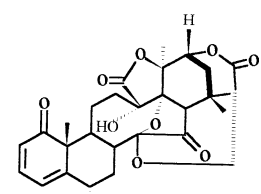

$\mathrm{OH}$

(28) fisalina $\underline{G}^{(32)}$

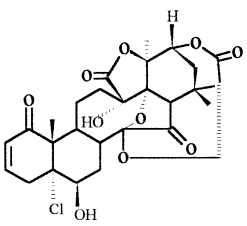

(30) fisalina $\underline{H}^{(34)}$
A estrutura da "fisalina $\underline{\mathrm{H}}$ " inicialmente descrita por Raw ${ }^{30}$ foi revisada por Kawai. et al. ${ }^{34}$, demonstrando tratar-se da estrutura (30).

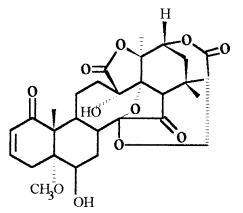

(31) fisalina $\underline{\underline{I}}^{(32)}$

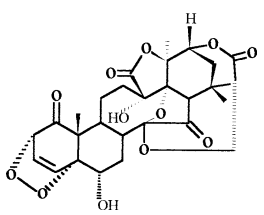

(33) fisalina $\underline{K}^{(32,35)}$

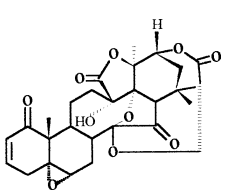

(32) fisalina $\underline{\mathbf{J}}^{(31)}$

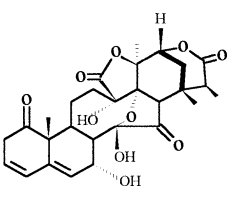

(34) fisalina $\underline{L}^{(36-38)}$ 


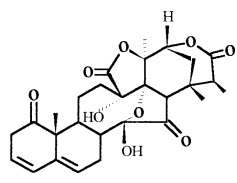

(35) fisalina $\underline{\mathbf{M}}^{(39)}$

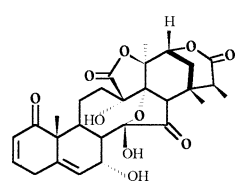

(37) fisalina $\underline{\mathrm{O}}^{(40,41)}$

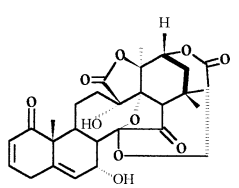

(36) fisalina $\underline{\underline{N}}^{(40,41)}$

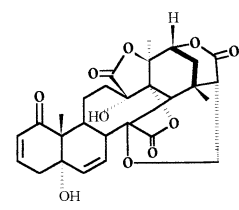

(38) fisalina $\underline{\underline{N}}^{(40,41)}$
A Fisalina $\underline{L}$, foi inicialmente descrita em $1995^{37}$, porém, somente foi definida sua estrutura (34) por Kawai e seus colaboradores $^{38}$ em 1996.

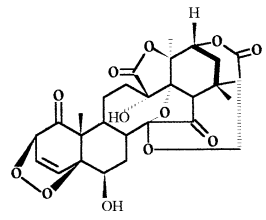

(39) fisalina $Q^{(35)}$

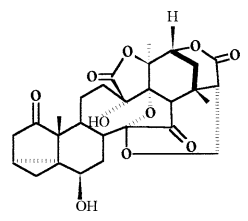

(41) fisalina $\underline{\underline{S}}^{(33)}$

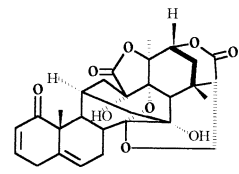

(40) fisalina $\underline{R}^{(33)}$

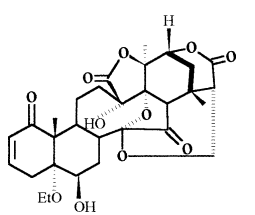

(42) 5- $\alpha$-etóxi-6- $\beta$-hidróxi-5,6-diidrofisalina $\underline{\mathbf{N}}^{(38)}$

As fisalinas possuem esqueletos relacionados aos anéis $\mathrm{A}$ e B, compreendendo os tipos ${ }^{2}$ :<smiles>CC1CCC2CC=CC(=O)C12C</smiles><smiles>CCC1CC=C2CC=CC(=O)C21C</smiles>

OlI

$\underline{\mathrm{d}}$<smiles>CC1CCC2CC=CC(=O)C2C1</smiles>

$\mathrm{OH}$

g
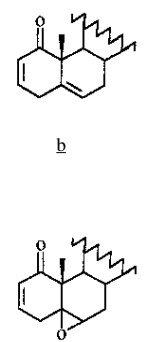

$\underline{\mathrm{e}}$

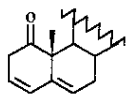

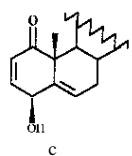

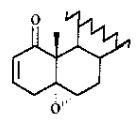

$\underline{\mathrm{f}}$

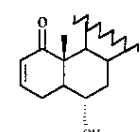

ö
Figura 2. Relação entre os anéis A e B nas fisalinas.

Existem exceções a estes nove exemplos acima ilustrados, pois as fisalinas $\underline{K}$ (33) e $\underline{Q}$ (39) portam um peróxido no anel $\mathrm{A}^{35}$ enquanto que na fisalina $\underline{\mathrm{S}}$ (41) não se observa nenhuma insaturação no primeiro anel, isto é, no anel A.
Este grupo de constituintes também mostram distinções em outros sítios da molécula como na ponte oxirânica C-14 e C27, ausente nas fisalinas $\underline{\mathrm{A}}$ (22), $\underline{\mathrm{C}}$ (24), $\underline{\mathrm{L}}$ (34), $\underline{\mathrm{M}}$ (35) e $\underline{\mathrm{O}}$ (37). A diferenciação entre estas cinco últimas estruturas dá-se na ligação entre os carbonos C-25 e C-27, que é metilênica para $\underline{\mathrm{A}}$ e $\underline{\mathrm{C}}$, e metílica (saturado) em $\underline{\mathrm{L}}, \underline{\mathrm{M}}$ e $\underline{\mathrm{O}}$.

À exceção das demais, a fisalina $\underline{\mathrm{P}}$ (38) possui um anel lactônico entre os carbonos 14 e 17, enquanto a fisalina $\underline{\mathrm{R}}$ (40), isolada de $P$. alkekengi var francheti, apresenta uma ponte entre os carbonos 11 e 15. Sua estrutura foi comprovada através da reação foto-induzida ${ }^{33}$ a partir da fisalina $\underline{B}$ (23).

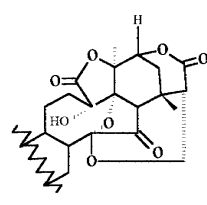

$\underline{3}$

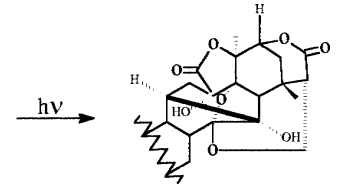

$\underline{\mathrm{R}}$
Esquema 4. Reação foto-induzida entre fisalina $\underline{B}$ e fisalina $\underline{R}$.

A fisalina $\underline{S}$ (41), caracteriza-se pela ausência dos sinais olefínicos no anel A; em contraposição apresenta sinais dos prótons metínicos com valores em campo alto, no $\mathrm{RMN}{ }^{1} \mathrm{H}$, indicando a presença de anel ciclopropano entre C-3 e C- $5^{33}$.

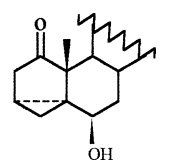

estrutura parcial de S

\section{- Quimiossistemática}

O gênero Physalis inclui cerca de cento e vinte espécies com caracteres herbáceos e hábitos perenes, que se distribuem pelas zonas temperadas do mundo principalmente nas Américas Central e $\mathrm{Sul}^{4}$.

O nome Physalis é oriundo do grego onde "physa" significa bolha ou bexiga, referindo-se ao cálice que encerra seus frutos, comestíveis na maioria das vezes.

Considerando o nível de oxidação biogenética, o gênero é o mais evoluído na família Solanaceae. Esta importante posição é devida à presença de metabólitos polioxigenados, os vitaesteróides, derivados do ergostano, com vinte oito átomos de carbono encerrando funções lactonas, epóxidos, enona. O sistema enzimático, nas plantas do gênero Physalis possui habilidade de oxidar o átomo de carbono do núcleo esteroidal e da cadeia lateral, com exceção aos carbonos C-8, C-9 e C-11 originando ampla variedade de estruturas químicas: fisalinas, vitafisalinas, ixocarpalactonas, acnistinas, dentre as demais. A família Solanaceae é caracterizada pela predominância destes vitaesteróides, sendo que Physalis ocupa lugar de destaque, dentre os doze gêneros.

Uma variável e extensa presença de constituintes químicos inclui flavonóides simples ou glicosilados (Campferol, quercetina, rutina, com uma, duas ou três unidades de açúcares $)^{48,49}$, esteróides ( $\beta$-sitosterol, estigmasterol, campestrol, 24-metileno-colesterol, dentre outros); ácidos graxos de cadeia linear $\left(\mathrm{C}_{6}\right.$ a $\left.\mathrm{C}_{24}\right)$, hidroxilados, epoxidados; carotenóides; ácido ascórbico e alcalóides ${ }^{50-52}$.

Variações estruturais ricas em funções oxigenadas, apresentando moléculas de açúcares e halogênios podem ser 
exemplificadas pelos vitaesteróides isolados de Physalis angulata $\mathrm{L}$., fisagulinas $\underline{\mathrm{C}} \mathrm{e} \underline{\mathrm{D}}^{53,54}(43-44)$ e fisalactona (45), esta última extraída de $P$. peruviana ${ }^{55}$.

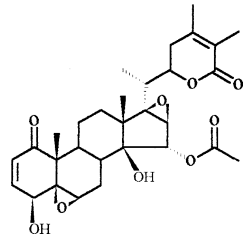

(43) fisangulina $\mathrm{C}$

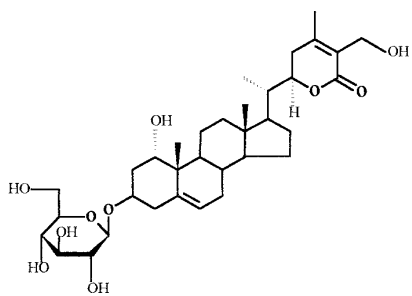

(44) fisangulina $D$

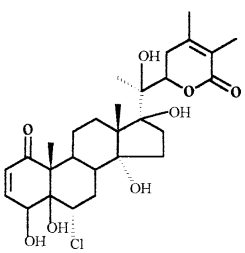

(45) fisalactona
A ocorrência de alcalóides no gênero Physalis está principalmente relacionada com a presença de derivados do tropano. A (+) fisoperuvina (46), seu isômero e o sal (+) N,N dimetilfisoperuvíneo (47), cujo anion é desconhecido, constituem o primeiro grupo biogeneticamente interessante de alcalóides tropânicos isolados a partir de raízes de Physalis peruviana ${ }^{56}$.

O conhecimento da biogênese do tropano indica que estes alcalóides não podem ser os intermediários, sendo provavelmente formados pela fusão da tropinona bicíclica através da eliminação do tipo Hoffmann e subseqüente redução da ligação dupla. A tigliodina (48), 3- $\beta$-tigloil-oxitropano, estão presentes na maioria dos membros do gênero Physalis ${ }^{47,57}$, juntamente com a figrina e anaferina (49 e 50), dentre os demais.<smiles>CN(C)[C@H]1CCCCC(=O)C1</smiles>

(46) fisopiruvina

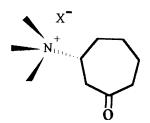

(47)(+) N,N-dimetilfisoperuvíneo

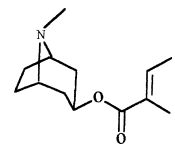

(48) tigliodina

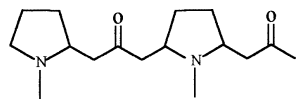

(49) figrina

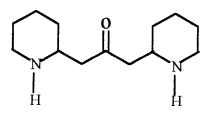

(50) anaferina

\section{ELUCIDAÇÃO ESTRUTURAL}

Os métodos espectroscópicos de ultravioleta, infravermelho, ressonância magnética nuclear $\left({ }^{1} \mathrm{H}\right.$ e $\left.{ }^{13} \mathrm{C}\right)$, espectrometria de massas, dicroismo circular e efeito Cotton são, sem dúvida, os recursos mais importantes e usuais na elucidação estrutural das substâncias presentes em Physalis ${ }^{3,4}$.

\section{Ultravioleta}

Os cromóforos presentes nos derivados do ergostano, com vinte e oito átomos de carbono, cognominados vitaesteróides possuem, em geral, grupos comuns que se constituem em: enona; $\delta$-lactona; $\alpha, \beta$-insaturada; $\gamma$-lactona.
As moléculas portadoras do sistema conjugado 2-en-1-ona, por exemplo, absorvem no ultravioleta próximo a $220 \mathrm{~nm}$, com uma absortividade molar alta. No caso da fisalina $\underline{G}(28)^{32}$, onde o anel A é biinsaturado, observa-se presença de outra dupla ligação entre os carbonos 4 e 5 estendendo a conjugação, formando, uma dienona homoanular (2,4-dien-1-ona) ocorrendo assim um efeito batocrômico cujo máximo de absorção está situado em $310 \mathrm{~nm}^{3,32}$.

A ausência destes cromóforos pode modificar as características espectrais. A saturação da ligação dupla na lactona não afeta a posição do $\lambda$ máximo porém, reduz o valor da absortividade molar, já a redução da dupla ligação da enona altera tanto os valores do $\lambda$ máximo quanto da absortividade ${ }^{3}$.

\section{Infravermelho}

As diferentes funções oxigenadas presentes neste grupo de esteróides modificados podem ser facilmente detectadas pela espectroscopia de infravermelho.

A despeito desta riqueza de informações o que se observa, na literatura, são os dados que se concentram e se limitam às absorções carboniladas das cetonas, enonas, lactonas $(\alpha$ e $\gamma)$ cujas bandas podem ocorrer entre as regiões 1790 a $1650 \mathrm{~cm}^{-1}$.

A fisalina D (25) por exemplo, apresenta estiramentos a $1792,1742,1665 \mathrm{~cm}^{-1} 30-32,45$, enquanto que a fisalina $\mathrm{L}(34)$ mostra espectro com bandas em 1770, 1740, $1720 \mathrm{~cm}^{-1}$.

Os seguintes valores, podem ser observados nas Fisalinas $\underline{\mathrm{D}} \mathrm{e} \underline{\mathrm{L}}^{36}$ :

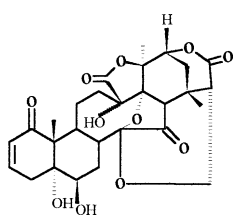

(25) fisalina $\underline{\mathrm{D}}^{(30-32)}$

$\gamma$-lactona - $1792 \mathrm{~cm}^{-1}$.

cetona no anel de 5 membros $-1742 \mathrm{~cm}^{-1}$.

cicloexanona - $1664 \mathrm{~cm}^{-1}$

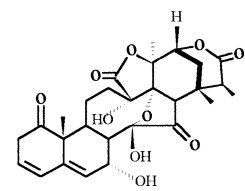

(34) fisalina $\underline{L}^{(36-38)}$

$\gamma$-lactona - $1770 \mathrm{~cm}^{-1}$.

cetona do anel dos cinco membros $-1740 \mathrm{~cm}^{-1}$

$\delta$-lactona - $1720 \mathrm{~cm}^{-1}$.

\section{Ressonância Magnética Nuclear - ${ }^{1} \mathrm{H}$ e ${ }^{13} \mathrm{C}$}

O incremento fornecido por esta técnica foi de enorme valia para aqueles que lidam com a química de produtos naturais. Iniciado em 1965 foi aumentado a partir da década de setenta onde pode ser observada, uma extensa lista de publicações referentes ao assunto ${ }^{84,85}$.

Os espectros de RMN de hidrogênio (RMN ${ }^{1} \mathrm{H}$ ) dos vitaesteróides tem características peculiares, como, por exemplo, o duplo par de duplo dublete do sistema enona e os acoplamentos com hidrogênios da posição 4 do anel A. Aquele par de absorções geralmente situa-se entre valores de 5,5 a 6,5 p.p.m. apresentando constantes de acoplamento entre 10 a 20 $\mathrm{Hz}$, dados comuns também às fisalinas. 


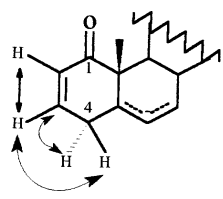

$\left\{\begin{array}{l}\text { dupla } \\ \text { epóxido } \\ \text { hidroxila } \\ \text { halogênio }\end{array}\right.$

estrutura parcial

dupla

epóxido

hidroxila

Correspondendo às posições 4, 5, 6 ou 7

halogênio ddd

$\mathrm{J}(3,2)$

$\mathrm{J}(3,4 \alpha)$
Diferentes substituições nos anéis A e B podem fornecer padrões que permitirão identificá-las pela espectroscopia de ressonância magnética nuclear.

As fisalinas exibem sinais típicos simples para suas três metilas angulares, C-19, C-21 e C-28 .

Enquanto que os vitanolídos, por exemplo, mostram sinais para cinco grupos metílicos, dois angulares C-18 e C-19; duas metilas vinílicas em C-27 e C-28, e uma secundária para o C-21, caso o carbono em C-20 não porte nenhum substituinte. Qualquer mudança no número destas metilas ${ }^{3,4}$ indicará, com ampla margem de segurança, substituições naqueles sítios ou mesmo modificações estruturais do esqueleto.

Nos últimos quatro anos a determinação estrutural dos vitaesteróides sofreu marcante desenvolvimento com auxílio das técnicas bidimensionais de RMN. Os deslocamentos químicos e a multiplicidade dos sinais, nos diversificados grupos destes derivados do ergostano, se encontram amplamente relatados na literatura ${ }^{3}$, cujos exemplos aqui apresentados são os mais recentes e atualizados ${ }^{33,34-38}$.

Os valores tabelados estão expressos em $\delta$, as constantes de acoplamento em $\mathrm{Hz}$ encontram-se entre parêntesis.

Os dados de RMN ${ }^{1} \mathrm{H}$, ora descritos (Tabelas 1-3), referem-se às fisalinas cujas estruturas são diversificadas no anel A e àquelas que não portam a ponte oxirânica entre os carbonos $\mathrm{C}-14$ e $\mathrm{C}-27$.

$\mathrm{O}$ artigo de revisão de Gottlieb e Kirson ${ }^{58}$ sobre RMN ${ }^{13} \mathrm{C}$ é bastante útil para aqueles que trabalham investigando substâncias deste grupo.

Os deslocamentos químicos dos carbonos de algumas fisalinas encontram-se ilustrados na Tabela 4.

\section{- Espectrometria de Massas}

Enquanto este método é muito útil para determinados gru$\operatorname{pos}^{86}$, tais como: vitanolídos e ixocarpalactonas, sua aplicação para elucidar as estruturas dos outros diferentes tipos de vitaesteróides é limitada.

O pico básico a $\mathrm{m} / \mathrm{z}, 125$ (ion) serve para diagnosticar os vitanolídos que portam uma $\delta$-lactona $\alpha, \beta$-insaturada correspondente a cisão $\mathrm{C}-20 / \mathrm{C}-22^{3}$ segundo esquema abaixo:

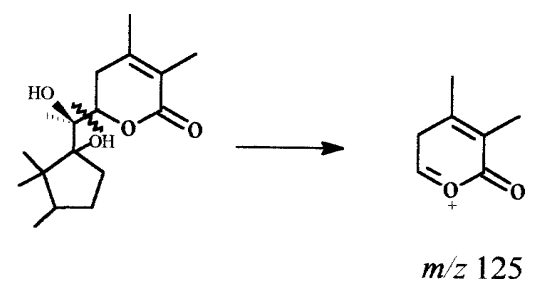

Esquema 5. Mecanismo proposto para formação do ion $\mathrm{m} / z 125$ pela cisão da ligação C-20/ C-22.

Como também este ion base $\mathrm{m} / \mathrm{z}, 125$ pode ser oriundo do anel A de vitaesteróides que possuam o sistema 5-hidróxi-2en-1-ona, conforme a seguinte fragmentação ${ }^{45}$ :

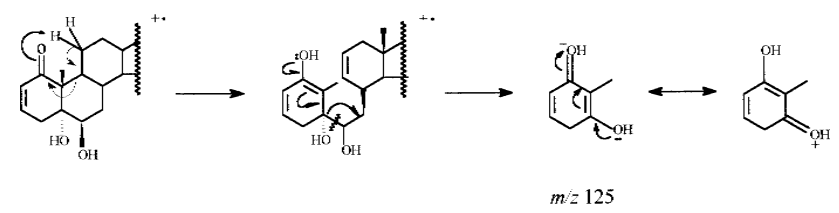

Esquema 6. Mecanismo proposto para o ion $\mathrm{m} / \mathrm{z} 125$ oriundo do anel $A$, dos vitaesteróides.

Então, o aparecimento do ion base $m / z, 125$ não pode ser considerado como evidência definitiva e inquestionável de um esqueleto de vitaesteróides portando uma $\delta$-lactona $\alpha, \beta$ insaturada ${ }^{82,83}$.

O padrão de fragmentação das fisalinas ainda não está bem definido pelos pesquisadores, embora Mulchandani et al. ${ }^{45}$, quando estudaram o espectro da fisalina $\underline{D}$ (25) e derivados, apresentaram algumas características em comum, como por exemplo, o ion $\mathrm{m} / \mathrm{z}$ 322. A gênese deste $\mathrm{m} / \mathrm{z} 322$ pode ser racionalizada pela fragmentação abaixo:

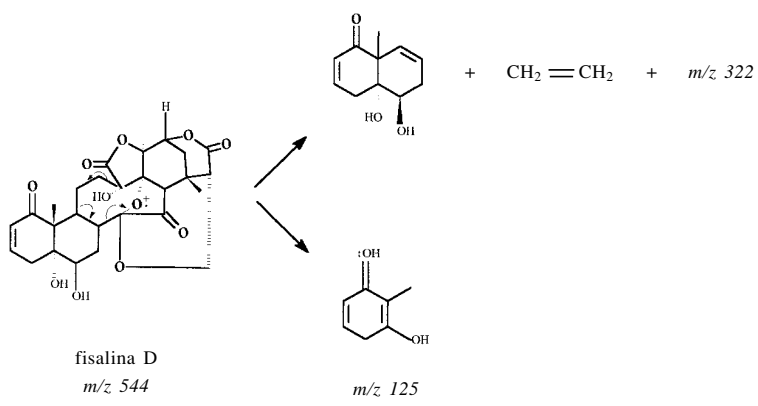

Esquema 7. Mecanismo de fragmentação da fisalina $D$ segundo Mulchandani et al ${ }^{45}$.

\section{Rotação Ótica}

Os valores obtidos com a rotação ótica também contribuem para a correta identificação e caracterização dos derivados esteroidais aqui relatados, fazendo assim parte do complexo de informações necessárias para elucidação estrutural.

As fisalinas apresentam os seguintes valores de rotação ótica:

\begin{tabular}{|c|c|c|}
\hline A & {$[\alpha]^{\mathrm{D}}$} & $-173^{\circ}(\mathrm{c} 0,2 \mathrm{EtOH})^{27,28}$ \\
\hline B & {$[\alpha]^{\mathrm{D}}$} & $-124^{\circ}(\mathrm{c} 0,54 \mathrm{EtOH})^{27}$ \\
\hline $\mathrm{C}$ & {$[\alpha]^{\mathrm{D}}$} & $-160^{\circ}\left(\mathrm{c} 0,145 \mathrm{Me}_{2} \mathrm{CO}\right)^{29}$ \\
\hline $\mathrm{D}$ & {$[\alpha]^{\mathrm{D}}$} & $-68^{\circ}(\mathrm{c} 1,34 \mathrm{MeOH})^{30,32}$ \\
\hline E & {$[\alpha]^{\mathrm{D}}$} & $-83^{\circ}\left(\mathrm{c} 0,5 \mathrm{CHCl}_{3}\right)^{33}$ \\
\hline $\mathrm{F}$ & {$[\alpha]^{\mathrm{D}}$} & $-20^{\circ}\left(\mathrm{c} 0,5 \mathrm{Me}_{2} \mathrm{CO}\right)^{31}$ \\
\hline G & {$[\alpha]^{\mathrm{D}}$} & $+17^{\mathrm{o}}\left(\mathrm{c} 0,5 \mathrm{Me}_{2} \mathrm{CO}\right)^{32}$ \\
\hline $\mathrm{H}$ & {$[\alpha]_{15}^{\mathrm{D}}$} & $-92^{\mathrm{o}}\left(\mathrm{c} 0,11 \mathrm{Me}_{2} \mathrm{CO}\right)^{34}$ \\
\hline I & {$[\alpha]^{\mathrm{D}}$} & $+12^{\mathrm{o}}\left(\mathrm{c} 0,5 \mathrm{Me}_{2} \mathrm{CO}\right)^{32}$ \\
\hline J & {$[\alpha]^{\mathrm{D}}$} & $-60^{\circ}(\mathrm{c} 0,5 \mathrm{Me} 2 \mathrm{CO})^{31}$ \\
\hline K & {$[\alpha]_{20}^{D_{2}}$} & $-224^{\circ}(\mathrm{c} 0,04 \mathrm{MeOH})^{35}$ \\
\hline $\mathrm{L}$ & {$[\alpha]_{24}^{D_{20}}$} & $-118^{\mathrm{o}}\left(\mathrm{c} 0,3 \quad \mathrm{Me}_{2} \mathrm{CO}\right)^{38}$ \\
\hline M & {$[\alpha]_{24}^{D_{24}^{24}}$} & $-106^{\mathrm{o}}\left(\mathrm{c} 0,34 \mathrm{Me}_{2} \mathrm{CO}\right)^{39}$ \\
\hline $\mathrm{N}$ & {$[\alpha]_{24}^{D_{24}^{24}}$} & $-124^{\mathrm{o}}\left(\mathrm{c} 0,14 \mathrm{Me}_{2} \mathrm{CO}\right)^{41}$ \\
\hline $\mathrm{O}$ & {$[\alpha]_{24}^{D_{24}}$} & $-115^{\mathrm{o}}\left(\mathrm{c} 0,1 \mathrm{Me}_{2} \mathrm{CO}\right)^{41}$ \\
\hline $\mathrm{P}$ & {$[\alpha]_{24}^{D_{2}}$} & $+54^{\circ}\left(\mathrm{c} 0,25 \mathrm{Me}_{2} \mathrm{CO}\right)^{42}$ \\
\hline $\mathrm{Q}$ & {$[\alpha]_{20}^{D_{2+}^{2+}}$} & $+38^{\circ}\left(\mathrm{c} 0,06 \mathrm{Me}_{2} \mathrm{CO}\right)^{35}$ \\
\hline R & {$[\alpha]_{15}^{D_{15}^{20}}$} & $-177^{\circ}\left(\mathrm{c} 0,13 \mathrm{Me}_{2} \mathrm{CO}\right)^{33}$ \\
\hline S & {$[\alpha]_{15}^{\mathrm{D}}$} & $-118^{\circ}\left(\mathrm{c} 0,08 \mathrm{Me}_{2} \mathrm{CO}\right)^{33}$ \\
\hline
\end{tabular}


Tabela 1. Dados de $\mathrm{RMN}^{1} \mathrm{H}$, em DMSO- $\mathrm{d}_{6}$, das fisalinas L e A.

\begin{tabular}{|c|c|c|c|c|c|}
\hline \multicolumn{3}{|c|}{ Fisalina $\mathrm{L}^{36}$} & \multicolumn{3}{|c|}{ Fisalina $\mathrm{A}^{3,44}$} \\
\hline $\mathrm{H}$ & $\delta$ & $\mathrm{J}$ & $\mathrm{H}$ & $\delta$ & $\mathrm{J}$ \\
\hline $2 \alpha$ & $2,68 \mathrm{dd}$ & $(20 ; 3)$ & 2 & $5,83 \mathrm{dd}$ & $(10 ; 2)$ \\
\hline $2 \beta$ & 3,47 brd & $(20)$ & - & - & - \\
\hline 3 & 5,88 brd & $(10 ; 3)$ & 3 & 6,93 ddd & $(10 ; 5 ; 2,5)$ \\
\hline 4 & $6,14 \mathrm{~d}$ & $(10)$ & $4 \alpha$ & $2,91 \mathrm{dd}$ & $(22 ; 5)$ \\
\hline 6 & 5,76 brd & $(5)$ & $4 \beta$ & 3,26 brd & $(22)$ \\
\hline 7 & $4,61 \mathrm{~m}$ & - & 6 & $5,69 \mathrm{dd}$ & $(6 ; 1,5)$ \\
\hline $7 \mathrm{OH}$ & $5,07 \mathrm{~d}$ & $(4)$ & 7 & 4,47 brt & (5) \\
\hline 8 & 2,05 brd & $(12)$ & $7 \mathrm{OH}$ & $5,00 \mathrm{~d}$ & $(4,5)$ \\
\hline 9 & $3,12 \mathrm{dd}$ & $(12 ; 9)$ & 8 & $1,81 \mathrm{dd}$ & $(12 ; 1,5)$ \\
\hline $11 \alpha$ & $1,53 \mathrm{dd}$ & $(16 ; 8)$ & 9 & $3,00 \mathrm{dd}$ & $(12 ; 9)$ \\
\hline $11 \beta$ & $1,15 \mathrm{~m}$ & - & $11 \mathrm{a}$ & $2,04 \mathrm{~m}$ & - \\
\hline $12 \alpha$ & $2,16 \mathrm{~m}$ & - & $11 \beta$ & $1,15 \mathrm{~m}$ & - \\
\hline $12 \beta$ & $1,93 \mathrm{dd}$ & $(16 ; 5)$ & $12 \alpha$ & 2,23 ddd & $(15 ; 12 ; 8)$ \\
\hline $13 \mathrm{OH}$ & $5,52 \mathrm{~s}$ & - & $12 \beta$ & $1,93 \mathrm{dd}$ & $(16 ; 5,5)$ \\
\hline $14 \mathrm{OH}$ & $6,85 \mathrm{~s}$ & - & $13 \mathrm{OH}$ & $5,60 \mathrm{~s}$ & - \\
\hline- & - & - & $14 \mathrm{OH}$ & $6,38 \mathrm{~s}$ & - \\
\hline 16 & $2,97 \mathrm{~s}$ & - & 16 & $3,08 \mathrm{~s}$ & - \\
\hline 19 & $1,16 \mathrm{~s}$ & - & $19 \mathrm{Me}$ & $1,02 \mathrm{~s}$ & - \\
\hline 21 & $1,71 \mathrm{~s}$ & - & $21 \mathrm{Me}$ & $1,71 \mathrm{~s}$ & - \\
\hline 22 & $4,57 \mathrm{~m}$ & - & 22 & $4,59 \mathrm{dd}$ & $(4 ; 2)$ \\
\hline 23 & 2,11 brd & $(15 ; 4)$ & $23 \alpha$ & $2,03 \mathrm{dd}$ & $(14 ; 4)$ \\
\hline 25 & $2,62 \mathrm{q}$ & (8) & $23 \beta$ & $2,06 \mathrm{dd}$ & $(14 ; 2)$ \\
\hline- & - & - & $27(\mathrm{E})$ & 5,59 brs & - \\
\hline 27 & $1,17 \mathrm{~d}$ & (8) & $27(\mathrm{Z})$ & $6,43 \mathrm{~s}$ & - \\
\hline 28 & $1,32 \mathrm{~s}$ & - & $28 \mathrm{Me}$ & $1,55 \mathrm{~s}$ & - \\
\hline
\end{tabular}

Tabela 2. Dados de $R M N^{1} H$, em DMSO- $d_{6}$, das fisalinas B e Q.

\begin{tabular}{|c|c|c|c|c|c|}
\hline \multicolumn{3}{|c|}{ Fisalina $\mathrm{B}^{40,43}$} & \multicolumn{3}{|c|}{ Fisalina $\mathrm{Q}^{35}$} \\
\hline $\mathrm{H}$ & $\delta$ & $\mathrm{J}$ & $\mathrm{H}$ & $\delta$ & $\mathrm{J}$ \\
\hline 2 & $5,80 \mathrm{dd}$ & $(10 ; 2)$ & 2 & $4,69 \mathrm{dd}$ & $(6 ; 1,5)$ \\
\hline 3 & 6,89 ddd & $(10 ; 5 ; 2)$ & 3 & $6,68 \mathrm{dd}$ & $(8 ; 6)$ \\
\hline $4 \alpha$ & $2,89 \mathrm{dd}$ & $(20 ; 5)$ & 4 & $6,76 \mathrm{dd}$ & $(8 ; 1,5)$ \\
\hline $4 \beta$ & 3,27 brd & $(20 ; 6)$ & $6 \alpha$ & $4,00 \mathrm{~m}$ & - \\
\hline 6 & 5,59 brd & (6) & $6 \mathrm{OH}$ & $5,24 \mathrm{~d}$ & $(3,5)$ \\
\hline $7 \alpha$ & $1,97 \mathrm{~m}$ & - & $7 \alpha$ & $1,74 \mathrm{~m}$ & - \\
\hline $7 \beta$ & $2,21 \mathrm{~m}$ & - & $7 \beta$ & $2,10 \mathrm{~m}$ & - \\
\hline 8 & $1,92 \mathrm{~m}$ & - & 8 & $2,30 \mathrm{dd}$ & $(12,5 ; 10,3)$ \\
\hline 9 & $2,95 \mathrm{dd}$ & $(11 ; 8)$ & 9 & 2,66 brt & $(10 ; 10)$ \\
\hline $11 \alpha$ & $2,18 \mathrm{~m}$ & - & $11 \alpha$ & $2,77 \mathrm{t}$ & $(15 ; 15)$ \\
\hline $11 \beta$ & $1,10 \mathrm{~m}$ & - & $11 \beta$ & $1,03 \mathrm{~m}$ & - \\
\hline $12 \alpha$ & $2,17 \mathrm{~m}$ & - & $12 \alpha$ & $1,85 \mathrm{~m}$ & - \\
\hline $12 \beta$ & $1,45 \mathrm{~m}$ & - & $12 \beta$ & $1,37 \mathrm{dd}$ & $(16,5 ; 10)$ \\
\hline $13 \mathrm{OH}$ & $6,28 \mathrm{~s}$ & - & $13 \mathrm{OH}$ & $6,43 \mathrm{~s}$ & - \\
\hline 16 & $2,86 \mathrm{~s}$ & - & 16 & $2,83 \mathrm{~s}$ & - \\
\hline $19 \mathrm{Me}$ & $1,09 \mathrm{~s}$ & - & $19 \mathrm{Me}$ & $1,31 \mathrm{~s}$ & - \\
\hline $21 \mathrm{Me}$ & $1,78 \mathrm{~s}$ & - & $21 \mathrm{Me}$ & $1,75 \mathrm{~s}$ & - \\
\hline 22 & $4,56 \mathrm{dd}$ & $(3 ; 2)$ & 22 & $4,55 \mathrm{~m}$ & - \\
\hline $23(\mathrm{~S})$ & $1,96 \mathrm{~m}$ & - & $23(\mathrm{R})$ & $2,08 \mathrm{~m}$ & $(\mathrm{R})$ \\
\hline $23(\mathrm{R})$ & $2,14 \mathrm{~m}$ & - & $23(\mathrm{~S})$ & $1,89 \mathrm{dd}$ & $(5-15 ; 2)$ \\
\hline 25 & 2,88 brd & (4) & 25 & $2,89 \mathrm{~d}$ & (4) \\
\hline $27(\mathrm{R})$ & $4,26 \mathrm{dd}$ & $(14 ; 4)$ & $27(\mathrm{R})$ & $4,26 \mathrm{dd}$ & $(13,5)$ \\
\hline $27(\mathrm{~S})$ & $3,60 \mathrm{dd}$ & $(14 ; 1)$ & $27(\mathrm{~S})$ & $3,59 \mathrm{~d}$ & $(13,5)$ \\
\hline $28 \mathrm{Me}$ & $1,16 \mathrm{~s}$ & - & $28 \mathrm{Me}$ & $1,15 \mathrm{~s}$ & - \\
\hline
\end{tabular}


Tabela 3. Dados de $R M N^{1} H$, em DMSO- $d_{6}$ das fisalinas $\mathrm{O}$ e $\mathrm{S}$.

\begin{tabular}{|c|c|c|c|c|c|}
\hline \multicolumn{3}{|c|}{ Fisalina $\mathrm{O}^{40}$} & \multicolumn{3}{|c|}{ Fisalina $\mathrm{S}^{33}$} \\
\hline $\mathrm{H}$ & $\delta$ & $\mathrm{J}$ & $\mathrm{H}$ & $\delta$ & $\mathrm{J}$ \\
\hline \multirow[t]{2}{*}{2} & $5,84 \mathrm{dd}$ & $(10,2)$ & $2 \alpha$ & 2,84 brd & $(17 ; 6)$ \\
\hline & - & - & $2 \beta$ & $1,92 \mathrm{~d}$ & $(17)$ \\
\hline 3 & 6,94 ddd & $(10 ; 5 ; 2,5)$ & 3 & $1,35 \mathrm{~m}$ & - \\
\hline $4 \alpha$ & $2,93 \mathrm{dd}$ & $(20 ; 5)$ & $4 \alpha$ & 0,70 ddd & $(6 ; 6,5 ; 05)$ \\
\hline $4 \beta$ & 3,27 brd & $(20)$ & $4 \beta$ & $0,21 \mathrm{dd}$ & $(6 ; 3,5)$ \\
\hline \multirow[t]{2}{*}{6} & $5,72 \mathrm{dd}$ & $(6 ; 1,5)$ & 6 & $3,18 \mathrm{~m}$ & - \\
\hline & & & $6 \mathrm{OH}$ & $4,50 \mathrm{~d}$ & (3) \\
\hline 7 & $4,51 \mathrm{dd}$ & $(6 ; 4,5)$ & $7 \alpha$ & 1,52 ddd & $(13 ; 10,5 ; 2,5)$ \\
\hline $7 \mathrm{OH}$ & $5,00 \mathrm{~d}$ & $(4,5)$ & $7 \beta$ & $2,17 \mathrm{dm}$ & (13) \\
\hline 8 & $1,88 \mathrm{~d}$ & $(12 ; 1,5)$ & 8 & $2,26 \mathrm{dt}$ & $(10,5 ; 1,5)$ \\
\hline 9 & $3,02 \mathrm{dd}$ & $(12 ; 9)$ & 9 & $2,58 \mathrm{t}$ & $(10,5 ; 14,5)$ \\
\hline $11 \alpha$ & $2,03 \mathrm{~m}$ & - & $11 \alpha$ & $1,67 \mathrm{~m}$ & - \\
\hline $11 \beta$ & $1,15 \mathrm{~m}$ & - & $11 \beta$ & $0,95 \mathrm{~m}$ & - \\
\hline $12 \alpha$ & $2,23 \mathrm{ddd}$ & $(16 ; 12 ; 8)$ & $12 \alpha$ & $1,8 \mathrm{~m}$ & - \\
\hline $12 \beta$ & $1,93 \mathrm{dd}$ & $(16 ; 5,5)$ & $12 \beta$ & $1,35 \mathrm{~m}$ & - \\
\hline $13 \mathrm{OH}$ & $5,57 \mathrm{~s}$ & - & $13 \mathrm{OH}$ & $6,63 \mathrm{~s}$ & - \\
\hline $14 \mathrm{OH}$ & $6,80 \mathrm{~s}$ & - & - & - & - \\
\hline 16 & $2,95 \mathrm{~s}$ & - & 16 & $2,85 \mathrm{~s}$ & - \\
\hline $19 \mathrm{Me}$ & $1,03 \mathrm{~s}$ & - & $19 \mathrm{Me}$ & $0,83 \mathrm{~s}$ & - \\
\hline 21 & $1,68 \mathrm{~s}$ & - & $21 \mathrm{Me}$ & $1,78 \mathrm{~s}$ & - \\
\hline 22 & 4,53 brd & (3) & 22 & $4,57 \mathrm{dd}$ & $(3,5 ; 2)$ \\
\hline $23 \alpha$ & $2,08 \mathrm{dd}$ & $(15 ; 4)$ & $23 \alpha$ & $2,10 \mathrm{dd}$ & (R 14,$5 ; 3,5)$ \\
\hline $23 \beta$ & 1,78 brd & $(15)$ & $23 \beta$ & $1,90 \mathrm{dt}$ & (s 14,5;2) \\
\hline 25 & $2,60 \mathrm{q}$ & $(7,5)$ & 25 & $2,90 \mathrm{~d}$ & $(4,5)$ \\
\hline \multirow[t]{2}{*}{$27 \mathrm{Me}$} & $1,15 \mathrm{~d}$ & $(7,5)$ & $27 \mathrm{R}$ & $3,60 \mathrm{~d}$ & (13) \\
\hline & - & - & $27 \mathrm{~S}$ & $4,26 \mathrm{dd}$ & $(13 ; 4,5)$ \\
\hline $28 \mathrm{Me}$ & $1,30 \mathrm{~s}$ & - & $28 \mathrm{Me}$ & $1,15 \mathrm{~s}$ & \\
\hline
\end{tabular}

Tabela 4. Dados de $\mathrm{RMN}{ }^{13} \mathrm{C}$ em DMSO- $\mathrm{d}_{6}$, das fisalinas $\mathrm{M}$ e N.

\begin{tabular}{|c|c|c|c|}
\hline \multicolumn{2}{|c|}{ Fisalina $\mathrm{M}^{39}$} & \multicolumn{2}{|c|}{ Fisalina $\mathrm{N}^{40}$} \\
\hline $\mathrm{C}$ & ppm & $\mathrm{C}$ & $\mathrm{ppm}$ \\
\hline 1 & 209,6 & 1 & 201,5 \\
\hline 2 & 39,5 & 2 & 126,9 \\
\hline 3 & 122,5 & 3 & 146,2 \\
\hline 4 & 126,4 & 4 & 32,2 \\
\hline 5 & 140,4 & 5 & 139,1 \\
\hline 6 & 128,0 & 6 & 125,5 \\
\hline 7 & 25,7 & 7 & 61,4 \\
\hline 8 & 40,8 & 8 & 44,2 \\
\hline 9 & 32,4 & 9 & 27,6 \\
\hline 10 & 55,1 & 10 & 52,7 \\
\hline 11 & 24,2 & 11 & 24,0 \\
\hline 12 & 26,1 & 12 & 25,7 \\
\hline 13 & 78,8 & 13 & 81,0 \\
\hline 14 & 101,2 & 14 & 106,3 \\
\hline 15 & 215,8 & 15 & 208,7 \\
\hline 16 & 54,0 & 16 & 52,9 \\
\hline 17 & 82,0 & 17 & 78,0 \\
\hline 18 & 171,7 & 18 & 171,7 \\
\hline 19 & 20,8 & 19 & 15,6 \\
\hline 20 & 82,3 & 20 & 80,3 \\
\hline 21 & 25,5 & 21 & 21,9 \\
\hline 22 & 76,4 & 22 & 76,2 \\
\hline 23 & 29,0 & 23 & 31,3 \\
\hline 24 & 34,3 & 24 & 30,7 \\
\hline 25 & 40,9 & 25 & 49,3 \\
\hline 26 & 172,2 & 26 & 167,4 \\
\hline 27 & 16,6 & 27 & 61,1 \\
\hline 28 & 18,0 & 28 & 24,3 \\
\hline
\end{tabular}

Tabela 5. Dados de RMN ${ }^{13} \mathrm{C}$, em DMSO- $\mathrm{d}_{6}$, das fisalinas $\mathrm{R}$ e K.

\begin{tabular}{rccc}
\hline \multicolumn{2}{c}{ Fisalina $\mathrm{R}^{40}$} & \multicolumn{2}{c}{ Fisalina $\mathrm{K}^{35}$} \\
\hline $\mathrm{C}$ & $\mathrm{ppm}$ & $\mathrm{C}$ & $\mathrm{ppm}$ \\
\hline 1 & 202,7 & 1 & 207,5 \\
2 & 127,1 & 2 & 77,5 \\
3 & 148,6 & 3 & 126,6 \\
4 & 32,1 & 4 & 141,5 \\
5 & 137,7 & 5 & 83,4 \\
6 & 124,4 & 6 & 64,8 \\
7 & 25,4 & 7 & 28,1 \\
8 & 43,2 & 8 & 37,5 \\
9 & 44,7 & 9 & 32,4 \\
10 & 51,4 & 10 & 48,1 \\
11 & 46,9 & 11 & 20,2 \\
12 & 34,7 & 12 & 24,6 \\
13 & 85,9 & 13 & 78,1 \\
14 & 112,0 & 14 & 105,9 \\
15 & 75,6 & 15 & 209,2 \\
16 & 49,6 & 16 & 53,9 \\
17 & 82,1 & 17 & 80,5 \\
18 & 173,8 & 18 & 171,8 \\
19 & 17,6 & 19 & 18,4 \\
20 & 81,9 & 20 & 80,2 \\
21 & 20,4 & 21 & 21,6 \\
22 & 75,6 & 22 & 76,3 \\
23 & 31,0 & 23 & 31,3 \\
24 & 31,3 & 24 & 30,4 \\
25 & 50,1 & 25 & 49,3 \\
26 & 168,6 & 26 & 167,1 \\
27 & 60,0 & 27 & 60,6 \\
28 & 28,3 & 28 & 24,4 \\
\hline
\end{tabular}




\section{Dicroismo Circular e Efeito Cotton}

Dados de Dicroismo Circular e Efeito Cotton são freqüentemente utilizados para informar a disposição de centros quirais, como também colaboram na determinação da configuração da fusão de anéis nos vitoesteróides, em especial, dos anéis A/B.

Os ergostanos com o sistema de conjugação 2-en-1-ona apresentam efeito Cotton em torno de $340 \mathrm{~nm}$ cujo sinal auxilia no reconhecimento da fusão $\mathrm{A} / \mathrm{B}$. Se o mesmo for positivo a junção é cis; se negativo a fusão é trans. Esta relação pode, contudo, apresentar exceções ${ }^{46}$.

As conformações dos vitaesteróides estão descritas extensivamente no artigo de Vasina, et $\mathrm{al}^{60}$.

Encontram-se, a seguir, os dados de DC para algumas fisalinas:

S $[\theta]_{304}-9.400 ;[\theta]_{296}-9.400 ;[\theta]_{224}-3.900^{33}$

$\mathrm{R}[\theta]_{328}-3.600 ;[\theta]_{260}+6.400 ;[\theta]_{227}-20.400 ;[\theta]_{208}-20.400^{33}$

$\mathrm{Q}[\theta]_{331}+26.500 ;[\theta]_{325}+29.400^{35}$

$\mathrm{P}[\theta]_{332}-6.200 ;[\theta]_{238}+12.500^{42}$

$\mathrm{K}[\theta]_{332}-33.400 ;[\theta]_{326}-35.200^{35}$

$\mathrm{H}[\theta]_{343}-5.300 ;[\theta]_{334}-5.700 ;[\theta]_{229}-13.800^{34}$

$\mathrm{D}[\theta]_{346} \Delta \varepsilon-2,13 ;[\theta]_{334} \Delta \varepsilon-1,61 ;[\theta]_{232} \Delta \varepsilon-3,28^{32}$

I $[\theta]_{346} \Delta \varepsilon-1,53 ;[\theta]_{334} \Delta \varepsilon-1,60 ;[\theta]_{234} \Delta \varepsilon-6,42^{32}$

$\mathrm{F}[\theta]_{338} \Delta \varepsilon+1,95 ;[\theta]_{352,5} \Delta \varepsilon-1,68^{31}$

J $[\theta]_{336} \Delta \varepsilon-1,86 ;[\theta]_{340} \Delta \varepsilon-1,79^{31}$

Acima estão representados exemplos de vitaesteróides cujos sinais positivo ou negativo do efeito Cotton, para absorções próximas a $340 \mathrm{~nm}$, informam sobre a configuração da junção dos dois primeiros anéis ${ }^{72,73}$.

É importante ressaltar, entretanto, que esta relação se inverte nos vitaesteróides quando a ligação dupla formando a enona está ausente ${ }^{46}$.

A anomalia observada no caso de vitaperuvina é atribuída por Trolow et al. ${ }^{6}$ ao efeito conformacional de três grupos hidroxilas contíguas, em C-4, C-5 e C-6 da molécula (j_a n $)$.

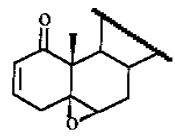

j

$\Delta \varepsilon_{340}+1,69$

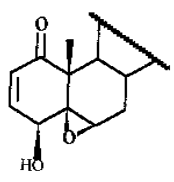

$\underline{\mathrm{k}}$

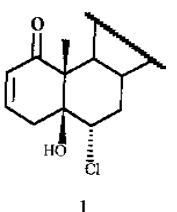

$\Delta \varepsilon_{342}+0,89$<smiles>C[C@H]1CC2CC3CC(CC31)C2(C)C</smiles>

$\underline{\mathrm{m}}$

$\Delta \varepsilon_{339}-0,67$

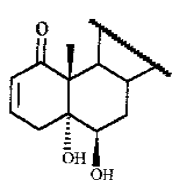

$\underline{\mathrm{n}}$

$\Delta \varepsilon_{336}-1,32$
Os vitaesteróides que portam um sistema $\delta$-lactona $\alpha, \beta$ insaturada apresentam Efeito Cotton positivo próximo à 240 $\mathrm{nm}$. Baseados nestas observações Snatzke et al. ${ }^{74}$ postularam para os vitanolídos a configuração 22R.
O único vitaesteróide natural cuja configuração é $22 \mathrm{~S}$ é o fisangulído (51), configuração esta proposta por Vasina et al ${ }^{75}$ em estudo detalhado dos derivados ergostânicos.

\section{ATIVIDADES BIOLÓGICAS}

As atividades biológicas dos vitaesteróides vem sendo descritas na literatura, e compreendem: antimicrobiana ${ }^{2,61,62}$, antiinflamatória, imunoduladora ${ }^{66}$ antitumoral $^{64,78}$, tripanossomicida $^{70,71,81}$ dentre outras ${ }^{1}$. Este amplo leque de atividades torna-se perfeitamente justificável em conseqüência da vasta diversificação de tipos estruturais que este grupo de esteróides é capaz de apresentar.

\section{Atividade Antimicrobiana}

Sem dúvida, a mais extensa divulgação cabe a witaferina $\underline{A}$ (9), o primeiro vitanolído isolado de Withania, cuja atividade antimicrobiana foi descrita em $1956^{61}$ antes mesmo de ter sua estrutura totalmente elucidada.

Em trabalho recente Tomassini et al. ${ }^{62}$, demonstraram atuação antibacteriana dos extratos etanólicos e aquosos de Physalis angulata L. quando ensaiados frente as cepas de Staphylococcus aureus e Escherichia coli.

\section{Atividade Antiinflamatória}

O fisangolído (51) e o epóxido vitanolído $\underline{\mathrm{D}}$ (52) apresentaram-se ativos como antiinflamatório nos modelos de reações tipo exudativa e proliferativa. Os resultados possibilitaram ou permitiram compará-los aos efeitos da hidrocortisona ${ }^{65}$.
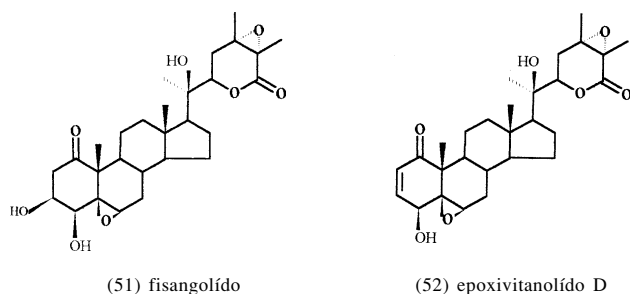

\section{Atividade Imunomoduladora}

Os estudos realizados com algumas espécies do gênero Physalis, i.e, $P$. angulata, $P$. pubescens, $P$. viscosa, permitiram comprovar a difundida e propalada ${ }^{1}$ resposta imunopositiva através de uma série de ensaios realizados in vitro e in vivo com material botânico proveniente das supra citadas espécies ${ }^{66}$.

$\mathrm{O}$ efeito in vitro pode ser constatado empregando transformações blastogênicas de linfócitos humanos enquanto que nas experiências in vivo os pesquisadores aplicaram os testes em camundongos imunizados com eritrócitos de Ramstd tratados, i.p., com doses que variaram entre 50 a $100 \mathrm{mg} / \mathrm{kg}$ das substâncias extraídas. Oito vitaesteróides, derivados do ergostano, apresentaram resultados positivos ${ }^{66}$.

O grupo de pesquisadores liderado por Chiang do Dept $^{\mathrm{o}}$ de Microbiologia e Imunologia da Faculdade de Medicina, Universidade de Cheng Kung, em Taiwan, em 1992, trabalhou com extratos e frações de $P$. angulata L. Descreveram aqueles pesquisadores que obtiveram acentuada resposta blastogênica com três substâncias do fracionamento. Os constituintes daquelas frações possuem atividade estimuladora nas células $\mathrm{B}$ e $\mathrm{T}$. As respostas dos anticorpos foram observadas em camundongos $\mathrm{BALB} / \mathrm{C}$ e $\mathrm{C}_{3} \mathrm{H} / \mathrm{HeJ}^{76}$.

Experiências por nós realizadas para avaliação imunofarmacológica de Physalis angulata L. $^{77}$ mostraram, no teste da incorporação de timidina [metil-3H], potente capacidade de induzir a proliferação de linfócitos in vitro. 


\section{Atividade Antitumoral}

A atividade antitumoral das fisalinas têm sido motivo de várias especulações e pesquisas. A fisalina $\underline{B}$ (23), por exemplo, apresentou-se citotóxica para células, da nasofaringe, 9 $\mathrm{KB}$, enquanto a $\underline{\mathrm{D}}$ (25) mostrou ser ativa para cepa $\mathrm{B}-16$ do mielocarcicoma ${ }^{63,64}$. Já a fisalina $\underline{A}$ (22) foi moderadamente ativa in vitro para células de tumor da cervix uterina, HeLa, entretanto as fisalinas $\underline{B}$ e $\underline{F}$ (23 e 27) apresentaram atividade superior a $\underline{\mathrm{A}}$ (22), sendo a $\underline{\mathrm{L}}$ (34) inativa.

Para os estudiosos da química de medicamentos, a busca da correlação estrutura-atividade tem sido o fundamento de numerosos trabalhos que permitem especular e entender, por exemplo, por que na ausência da conjugação tipo 2-en-1-ona a fisalina $\underline{L}(34)$, se torna inativa ${ }^{36}$.

A atividade antineoplásica de fisalina $\underline{F}$ (27) foi evidenciada e comprovada quando foram ensaiadas, in vitro, cinco linhagens de células cancerígenas humanas: HA 22 T (hepatoma); HeLa (cervix uterina); KB (nasofaringe); Colo-205 (colon) e Calu-1 (pulmão), os melhores resultados foram aqueles obtidos com as cepas HeLa e HA $22 \mathrm{~T}^{67}$.

A fisalina $\underline{F}$ (27) também mostrou efeito antitumoral in vivo contra células P 388 na leucemia linfocítica em camundongos ${ }^{67}$.

A partir de $P$. angulata foi isolada a substância vitangulatina A (53), um novo vitanolído capaz de atuar na clivagem do DNA através do mecanismo de indução enzimática tipo Topoisomerase II - DNA ${ }^{68,69}$.

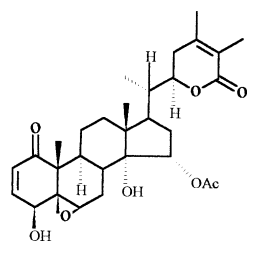

(53) vitangulatina $\mathrm{A}$

\section{Atividade Tripanossomicida}

No país, a Doença de Chagas afeta milhares de brasileiros apresentando até hoje prevalência e incidência elevadas, induzindo assim grupos de pesquisadores a trabalharem na resolução do problema.

Nesta década duas publicações, abaixo referenciadas, sobre as tripanossomiases apontam resultados promissores no combate a esta endemia que assola as populações dos trópicos.

A literatura pertinente a Physalis angulata L., sobre este tópico, relata dados de Kastelein e Camargo ${ }^{70}$ que pesquisando os frutos desta planta encontraram a tripanossomatina, uma enzima oriunda de tripanossomídeos flagelados, fato este relevante para a compreensão da biogênese do parasito e sua concomitante atuação.

Extratos de P. angulata L. testados frente a Trypanossoma brucei rhodesiense (doença do sono), por um grupo de pesquisadores suíços e africanos, apresentaram valores de $\mathrm{IC}_{50}$ abaixo de $1 \mathrm{mg} / \mathrm{mL}$ mostrando assim um índice de seletividade superior a produtos farmacêuticos aplicados como tripanossomicidas ${ }^{71}$.

Experiências realizadas nos laboratórios de Farmacologia Aplicada de Far-Manguinhos/FIOCRUZ comprovam a atividade de $P$. angulata L., uma vez que nos ensaios in vitro com cepas de $T$. cruzi, houve morte do parasita em percentuais de $95 \%$ à $100 \%^{81}$.

\section{CONSIDERAÇÕES GERAIS}

Os exemplos de vitaesteróides, oriundos do gênero Physalis, apontados na literatura cujos os dados de ensaios e testes biológicos envolvem as atividades aqui relacionadas indicam respostas significativas para o incremento das pesquisas com este grupo de substâncias esteroidais.

No campo da fisiologia vegetal, foram efetuados estudos de espécies clonais selvagens e cultivadas. Os resultados permitiram constatar que houve reprodução das fisalinas $\underline{B}(23), \underline{D}$ (25), $\underline{\mathrm{G}}$ (28), $\underline{\mathrm{H}}$ (30) e $\underline{\mathrm{L}}(34)$ nos calos de P. angulata. ${ }^{79} \mathrm{~A}$ presença daquelas substâncias foi comprovada através dos dados obtidos por HPLC e espectrometria de massas. ${ }^{80}$ Estes resultados indicam a aplicabilidade no atendimento ao parque industrial do país para produção da espécie $P$. angulata, bem como, atende aos requisitos da biodiversidade no combate ao desenfreado extrativismo do reino vegetal brasileiro.

A atividade microbiológica pesquisada revelou que extratos etanólicos da cápsula do fruto e folhas de Physalis angulata L. foram ativos frente às cepas patogênicas de $E$. coli e $S$. aureus ${ }^{66}$.

As evidências descritas, nesta revisão, mostram a importância dos vitaesteróides como um enorme campo para estudos e buscas de novos e eficazes medicamentos.

\section{AGRADECIMENTOS}

As autoras agradecem a FAPERJ, CNPq, Far-Manguinhos/ FIOCRUZ pelos financiamentos que permitiram as realizações descritas neste trabalho.

\section{REFERÊNCIAS}

1. Purushothaman, K. K.; Vasanth, S.; J. Scient. Ind. Res. 1988, 47, 326.

2. Glotter, E.; Nat. Prod. Rep. 1991, 8, 415.

3. Ray, B. A.; Gupta, M.; in "Progress in the Chemistry of Organic Natural Products”, Spring Verlag; New York Wien, 1994, p. 1.

4. Vasina, O. E.; Maslennikova, V. A.; Abubakirov, N.K.; Khim. Prir. Soedin. 1986, 3, 263.

5. Srivastava, C.; Siddiqui, I. R.; Singh, J.; Tiwari, H. P.; J. Ind. Chem. Soc. 1992, 69, 111.

6. Chen, Z. L.; Wang, B. D.; Chen, M. Q.; Tetrahedron Lett. 1987, 28, 1673.

7. Chen, Z. L.; Wang, B. D.; Shen, J. H.; Phytochemistry 1988, 27, 2999.

8. Velde, V. V.; Lavie, O.; Budhiraja, R. D.; Sudhir, S.; Garg, K. N.; Phytochemistry 1983, 22, 2253.

9. Sinha, S. C.; Ali, A.; Bagchi, A.; Sahai, M.; Ray, A. B.; Planta Medica 1987, 53, 55.

10. Lavie, D.; Glotter, E.; Shvo, Y.; J. Chem. Soc. 1965, 7517.

11. a) Basey, K.; Mc Graw., B. A.; Wooley, J. G.; Phytochemistry 1992, 31, 4173. b) Barata, L.; Mors, W. B.; Kirson, I.; Lavie, D; An. Acad. Brasil. Ciênc. 1970, 42, 401.

12. Glotter, E.; Kirson, I.; Abraham, A.; Subramanian, S. S.; Sethi, P. D.; J. Chem. Soc. Perkin I, 1975, 1370.

13. Sahai, M.; Kirson, I.; J. Nat. Prod. 1984, 47, 527.

14. Sinha, S. C.; Ray, A. B.; Oshima, Y.; Bagchi, A.; Hikino, H.; Phytochemistry 1987, 26, 2115.

15. Sinha, S. C.; Ray, A. B.; J. Ind. Chem. Soc. 1988, 65, 740.

16. Kirson, I.; Zaretskii, K.; Glotter, E.; J. Chem. Soc., Perkin I 1976, 1244.

17. Usu Billaga, A.; De Castellano, G.; Zabel, V.; Watson, W. H.; J. Chem. Soc., Chem. Commun. 1980, 854.

18. Shingu, K.; Marubayashi, N.; Ueda, I.; Yahart, S.; Nohara, T.; Chem. Pharm. Bull. 1990, 38, 1107.

19. Glotter, E.; Kirson, I.; J. Nat. Prod. 1981, 44, 633.

20. Kirson, I.; Cohen, A.; Greenberg, M.; Gottlieb, H. E.; Glotter, E.; Varenne, P.; Abraham, A.; J. Chem. Soc. Perkin I 1980, 531.

21. Hirayama, M.; Gamoh, K.; Ikekawa, N.; Tetrahedron Lett. 1982, 4725.

22. Gottlieb, H. E.; Kirson, I.; Glotter, E.; Ray, A. B.; Sahai, M.; Ali, A.; J. Chem. Soc. Perkin I 1980, 2700. 
23. Sahai, M.; Gottlieb, H. E.; Ray, A. B.; Ali, A.; Glotter, E.; Kirson, I.; J. Chem. Res. 1982, 346.

24. Glotter, E.; Kirson, I.; Lavie, D.; Abraham, A.; in Bioorganic Chemistry van Tamelen E.E., ed. New York, Academic Press, 1978, p.57.

25. Kawai, M.; Ogura, T.; Butsugan, Y.; Taga, T.; Hayashi, H.; Tetrahedron 1991, 47, 2103.

26. Kawai, M.; Matsumoto, A.; Makino, B.; Mori, H.; Ogura, T.; Butsugan, Y.; Ogawa, K.; Hayashi, M.; Bull. Chem. Soc. Jpn. 1993, 66, 1299.

27. Matsuura, T.; Kawai, M.; Nakashima, R.; Butsugan, Y.; J. Chem. Soc. C. 1970, 664.

28. Kawai, M.; Taga, T.; Miwa, Y.; Butsugan, Y.; J. Cryst. Spect. Res. 1992, 22, 131.

29. Kawai, M.; Matsuura, T.; Tetrahedron 1970, 26, 1743.

30. Row, L. R.; Sarma, N. S.; Matsuura, T.; Nakashima, R.; Phytochemistry 1978, 17, 1641.

31. Row, L. R.; Sarma, N. S.; Reddy, K. S.; Matsuura, T.; Nakashima, R.; Phytochemistry 1978, 17, 1647.

32. Row, L. R.; Reddy, K. S.; Sarma, N. S.; Matsuura, T.; Nakashima, R.; Phytochemistry 1980, 19,1175.

33. Makino, B.; Kawai, M.; Kito, K.; Yamamura, H.; Butsugan, Y.; Tetrahedron 1995, 51,12529.

34. Kawai, M.; Makino, B.; Ogura, T.; Nakanishi, M.; Yamamura, H.; Butsugan, Y,; J. Nat. Prod. 1995, 58, 1668.

35. Makino, B.; Kawai, M.; Iwata, Y.; Yamamura, H.; Butsugan, Y.; Ogawa, K.; Hayashi, M.; Bull. Chem. Soc. Jpn. 1995, 68, 219.

36. Kawai, M.; Matsuura, T.; Kyuno, S.; Matsuki, H.; Takenaka, M.; Katsuoka, T.; Butsugan, Y.; Saito, K.; Phytochemistry 1987, 26, 3313.

37. Sen, G.; Pathak, H.D.; Phytochemistry 1995, 39, 1224.

38. Kawai, M.; Makino, B.; Yamamura, H.; Butsugan, Y.; Phytochemistry 1996, 43, 661.

39. Kawai, M.; Ogura, T.; Nakanishi, M.; Matsuura, T.; Butsugan, Y.; Mori, Y.; Harada, K.; Suzuki, M.; Bull. Chem. Soc. Jpn. 1988, 61, 2696.

40. Kawai, M.; Ogura, T.; Makino, B.; Matsumoto, A.; Yamamura, H.; Butsugan, Y.; Hayashi, H.; Phytochemistry 1992, 31, 4299.

41. Kawai, M.; Ogura, T.; Matsumoto, A.; Butsugan, Y.; Hayashi, M.; Chem. Express. 1989, 4, 97.

42. Kawai, M.; Matsumoto, A.; Makino, B.; Mori, H.; Ogura, T.; Butsugan, Y.; Ogana, K.; Hayashi, M.; Bull.Chem. Soc. Jpn. 1993, 66, 1299.

43. Matsuura, T.; Kawai, M.; Tetrahedron Lett. 1969 , 22, 1765

44. Kawai, M.; Tago, T.; Miwa, Y.; Butsugan, Y.; J. Cryst. Spectrosc. Res. 1992, 22, 131.

45. Mulchandani, N. B.; Iyer, S. S.; Badheka, L. P.; Planta Medica 1979, 37, 268.

46. Trolow, F.; Ray, A. B.; Sahai, M.; Glotter, E.; Gottlieb, H. E.; Kirson, I.; J. Chem. Soc. Perkin I 1981, 1029.

47. Basey, K.; McGraw, B. A.; Wooley, J. G.; Phytochemistry 1992, 31, 4173.

48. Elliger, C. A.; Easy, J. A.; Wais, J. A. C.; Biochem. System. Ecol. 1992, 20, 268.

49. Sahai, M.; Neogi, P.; J. Ind. Chem. Soc. 1984, 61, 171.

50. Rao, T. C.; Lakshminarayana, G.; Prasad, N. B. L.; Rao, S. J. M.; Azumodin, G.; Ramayya, D. A.; J. Am. Oil Chem. Soc. 1984, 61, 1472.

51. Badami, R. C.; Thakkar, J.; Fette Seifen Anstrichmittel 1984, 86, 115

52. Mahna, S. K.; Singh, D.; Ind. J. Pharm. 1974, 36, 138.

53. Shingu, K.; Yahara, S.; Nohara, T.; Okabe, H.; Chem. Pharm. Bull. 1992, 40, 2088.

54. Shingu, K.; Manibayashi, N.; Ueda, I.; Yamara, S.; Nohara, T.; Chem. Pharm. Bull. 1991, 39, 1591.
55. Nittala, S.; Frolow, F.; Lavie, D.; J. Chem. Soc., Chem. Comnum. 1981, 178.

56. Sahai, M.; Ray, A.; J. Org. Chem. 1980, 45, 3265.

57. Beresford, P.J.; Wooley, J.G.; Phytochemistry 1974, 43,2143

58. Gottlieb, E.; Kirson, I.; Org. Magn. Res. 1981, 16, 20.

59. Pelletier, S. W.; Mody, N. V.; Nowacki, J.; Bhattacharya, J.; J. Nat. Prod. 1979, 42, 512.

60. Moiseeva, G. P.; Vasina, O. E.; Abubakirov, N. K.; Khim. Prir. Soedin. 1990, 3, 371.

61. Kurup, P. A.; Curr. Sci. India 1956, 25, 57.

62. Tomassini, T. C. B.; Silva, M. T. G.; Sanches, E. G.; Ribeiro, I. M.; Boll. Chim. Farm. 1997, 136, 154.

63. Vasina, O. E.; Maslenmikova, V. A.; Abubakirov, N. K.; Khim. Prir. Soedin. 1986, 22, 263.

64. Mikail, D. A.; Abranison, D.; Tyson, R. L.; Chang, C. J.; McLauglin, J. L.; Peck, G.; Cassady, J. M.; J. Nat. Prod. 1981, 44, 591 .

65. Syrov, V. N.; Khusbaktove, Z. A.; Vasina, O. E.; Khim. Farm. Zh. 1989, 23, 610, C.A. III, 166955 a (1989).

66. Sakhilov, A. D.; Syrov, Y. N.; Usmanova, A. S.; Khushbaktova, Z. A.; Vasina, O. E.; Abubakirov, N. K.; Dokl. Akad. Nauk UzSSR 1990, 1, 43. C.A. 116:362h (1990).

67. Chiang, H. C.; Jaw, S. M.; Chen, C. F.; Kan, W. S.; Anticancer Res. 1992, 12, 837.

68. Juang, J. K.; Huang, H. W.; Chen, C. M.; Liu, H. J.; Biochem. Biophys. Res. Commum. 1989, 59, 1128.

69. Chen, C. M.; Chen, Z. T.; Hiresh, C. H.; Li, W. S.; Wen, S. Y.; Heterocycles 1990, 31, 1371.

70. Kastelein, P.; Camargo, E. P.; Mem. Inst. Oswaldo Cruz 1990, 85, 413.

71. Freibughauss, F.; Kaminsky, R.; Nkunga, M. H.; Brun, R.; J. Ethnopharmacol. 1996, 55, 1.

72. Glotter, E.; Kirson, I.; Abraham, A.; Lavie, D.; Tetrahedron 1973, 29, 1353.

73. Fajardo, V.; Podesta, F.; Shamma, M.; Freyer, A. J.; J. Nat. Prod. 1991, 54, 554.

74. Snatzke, G.; Schwang, H.; Welzel, P.; in "Some Newer Physical Methods in Structural Chemistry", Bonnet; R.; David, J. G. ed. London, 1967, p. 159.

75. Vasina, O. E.; Abdullaev, N. O.; Abubakirov, N. K.; Khim. Prir. Soedin. 1990, 26, 366.

76. Lin, Y. S.; Chiang, H. C.; Kan, W. S.; Hone, E.; Shih, S. J.; Won, M. H.; Am. J. Chinese Med. 1992, 20 , 233.

77. Carvalho, M. V.; Ribeiro-dos-Santos, R.; Tomassini, T.C.B.; Henriques, M.G.M.O.; XII Reunião Anual da Federação de Sociedades de Biologia Experimental, FeSBE, Caxambú, MG, 1997, V.I., 111.

78. Chiang, H. C.; Jaw, S. M.; Chen, P. M.; Anticancer Res. 1992, 12,1155

79. Vasconcellos, A. G.; Esquibel, M. A.; Ribeiro, I. M.; Tomassini, T. C. B.; I Encontro Internacional das Plantas Aromáticas e Medicinais, Conimbriga, Ansião, Portugal, abril, 1998, vol. I, 104.

80. Mazzei, J. L.; Ribeiro, I. M.; Silva, A. I. F.; Barbi, N. S.; Xavier, D. D.; Tomassini, T. C. B.; 7th Latin American Congress on Chromatography and Related Techniques, COLACRO VII, Águas de São Pedro, São Paulo, 1998, 133.

81. Ribeiro-dos-Santos, R.; Soares, R.; Ferreira, E. F.; 1998, resultados não publicados.

82. Ray, A . B. M.; Sahai, M.; Das, B. C.; J. Ind. Chem. Soc. 1978, 55, 1175

83. Fajardo, V.; Freyer, A. J.; Minard, R. D.; Shamma, M.; Tetrahedron 1987, 43, 3875.

84. Dorome, A. E .; Nat. Prod. Rep. 1989, 6, 111.

85. Williamson, M. P.; Nat. Prod. Rep. 1993, 10, 207.

86. Baldwin, M. A .; Nat. Prod. Rep. 1995, 12, 33. 